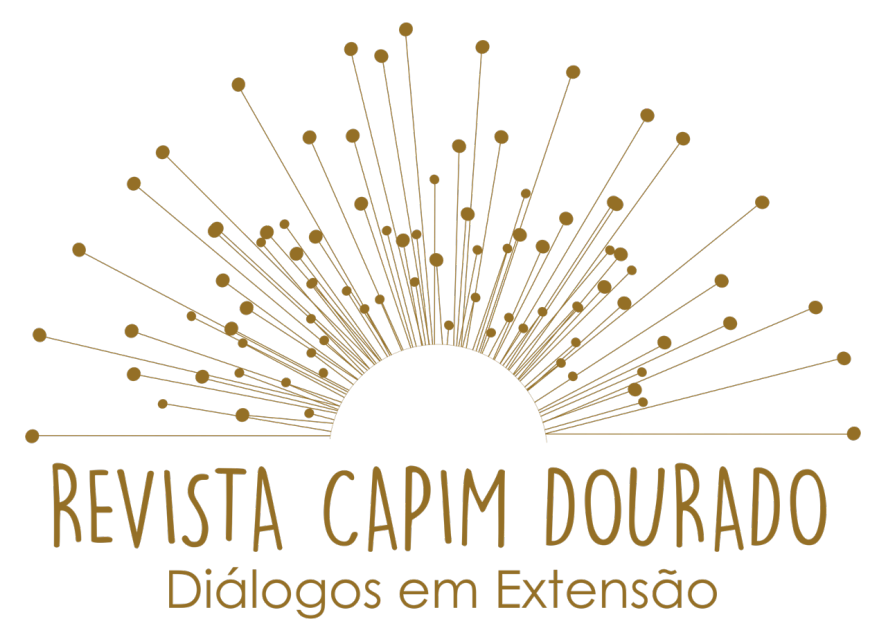

ISSN n² 2595-7341

Vol. 3, n. 1, Janeiro-Abril, 2020

DOI: http://dx.doi.org/10.20873/uft.2595-7341.2020v3n1p31

\title{
PROJETO UFT ALÉM DOS MUROS
}

UFT PROJECT BEYOND THE WALLS

PROYECTO UFT MÁS ALLÁ DE LAS PAREDES

\author{
Dhayna Oliveira Sobral ${ }^{1}$ \\ Kamila Veloso Silva² \\ Luiz Evandro de Melo Junior ${ }^{3}$ \\ Evandro de Luiz ${ }^{4}$ \\ Tábitha Bueno Akemi Ogawa ${ }^{5}$ \\ Abraham Giraldo Damian Zuniga ${ }^{6}$
}

\section{RESUMO}

O Programa de Educação Tutorial (PET) de Engenharia de Alimentos da Universidade Federal do Tocantins tem como um dos objetivos realizar atividades de extensão que possam integrar o meio acadêmico com a comunidade externa. Um dos projetos é a consultoria que está sendo realizada no Lar Batista F.F. Soren. Esse projeto consiste em aplicar o conhecimento técnico de boas práticas de manipulação e controle de qualidade a fim de evitar desperdícios e contaminação. A ideia foi originada com interesse em proporcionar essa oportunidade para essa instituição filantrópica localizada em Luzimangues distrito de Porto Nacional-TO, uma vez que se trata de uma instituição sem fins

\footnotetext{
${ }^{1}$ Universidade Federal do Tocantins (UFT). E-mail: dhay sobral@outlook.com.

2 Universidade Federal do Tocantins (UFT). E-mail: kamilaveloso2014@hotmail.com.

3 Universidade Federal do Tocantins (UFT). E-mail: luizjr melo@hotmail.com.

${ }^{4}$ Universidade Federal do Tocantins (UFT). E-mail: evandroluiz@gmail.com.

${ }^{5}$ Universidade Federal do Tocantins (UFT). E-mail: tabitha.ogawa@gmail.com.

${ }^{6}$ Universidade Federal do Tocantins (UFT). E-mail: abraham@uft.edu.br.
} 


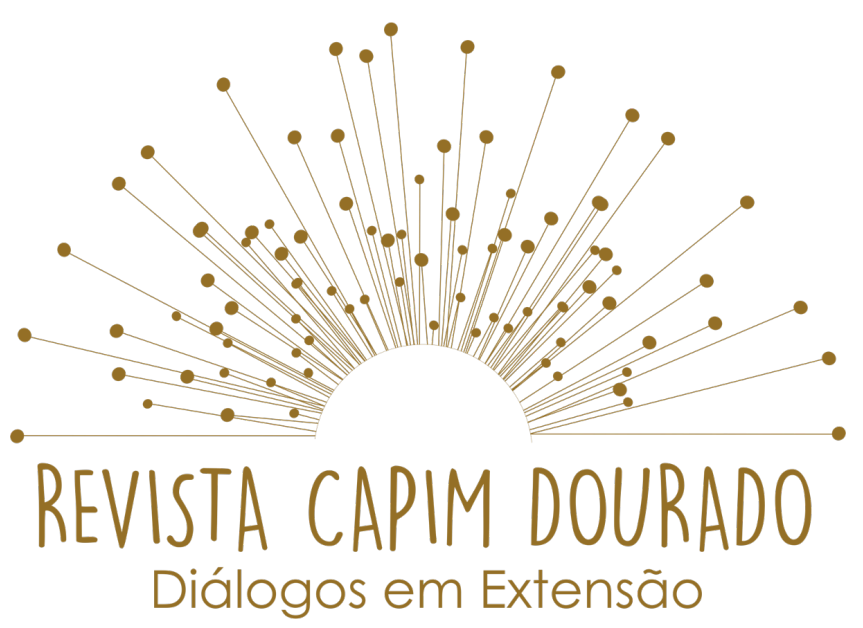

ISSN n² 2595-7341

Vol. 3, n. 1, Janeiro-Abril, 2020

DOI: http://dx.doi.org/10.20873/uft.2595-7341.2020v3n1p31

lucrativos como forma de colocar em prática os conhecimentos adquiridos durante o curso ajudando quem precisa. O projeto foi bem aceito, trazendo uma grande realização para o grupo, foi decido que o projeto terá continuidade, podendo ser realizado em longo prazo e com viabilidade para outros.

PALAVRAS-CHAVE: Consultoria. Social. Controle de qualidade. Boas práticas.

\section{ABSTRACT}

One of the objectives of the Food Engineering Tutorial Education Program (PET) at the Federal University of Tocantins is to carry out extension activities that can integrate the academic environment with the external community. One of the projects is the consultancy that is being carried out at Lar Batista F.F. Soren. This project consists of applying the technical knowledge of good practices in handling and quality control in order to avoid waste and contamination. The idea was originated with an interest in providing this opportunity for this philanthropic institution located in Luzimangues district of Porto Nacional-TO, since it is a nonprofit institution as a way of putting into practice the knowledge acquired during the course helping those in need. The project was well accepted, bringing great achievement to the group, it was decided that the project will continue, being able to be carried out in the long term and with feasibility for others.

KEYWORDS: Consulting. Social. Quality control. Good habits.

\section{RESUMEN}

Uno de los objetivos del Programa de Educación Tutorial en Ingeniería de Alimentos (PET) de la Universidad Federal de Tocantins es realizar actividades de extensión que puedan integrar el entorno académico con la comunidad externa. Uno de los proyectos es la consultoría que se está llevando a cabo en Lar Batista F.F. Soren. Este proyecto consiste en aplicar los conocimientos técnicos de buenas 


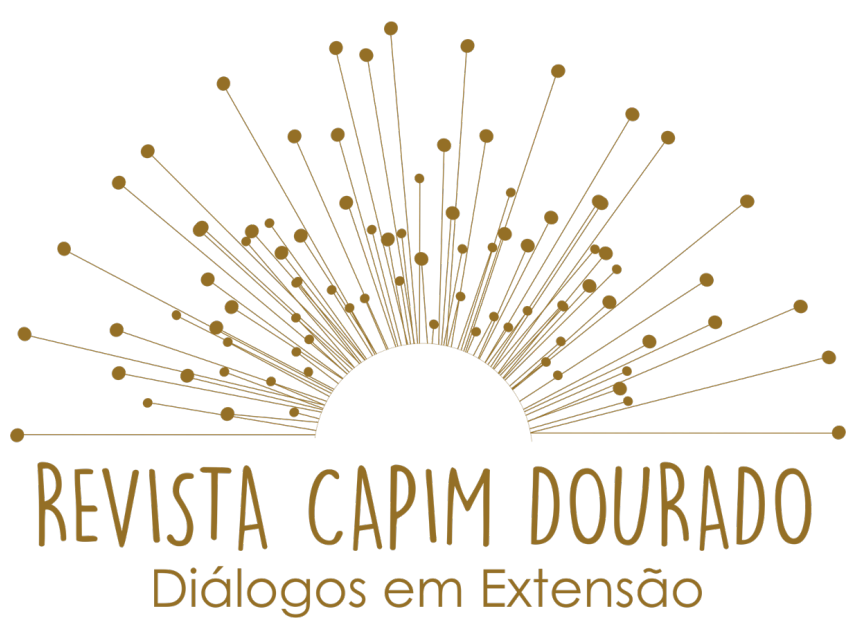

ISSN n² 2595-7341

Vol. 3, n. 1, Janeiro-Abril, 2020

DOI: http://dx.doi.org/10.20873/uft.2595-7341.2020v3n1p31

prácticas en el manejo y control de calidad con el fin de evitar residuos y contaminación. La idea nació con el interés de brindar esta oportunidad a esta institución filantrópica ubicada en el distrito de Luzimangues de Porto NacionalTO, ya que es una institución sin fines de lucro como una forma de poner en práctica los conocimientos adquiridos durante el curso ayudando a los necesitados. . El proyecto fue bien aceptado, trayendo gran logro al grupo, se decidió que el proyecto continuará, pudiendo llevarse a cabo en el largo plazo y con factibilidad para otros.

PALABRAS CLAVE: Consultoría. Social. Control de calidad. Buenas practicas.

Recebido em: 01.10.2019. Aceito em: 09.10.2019. Publicado em: 01.01.2020. 


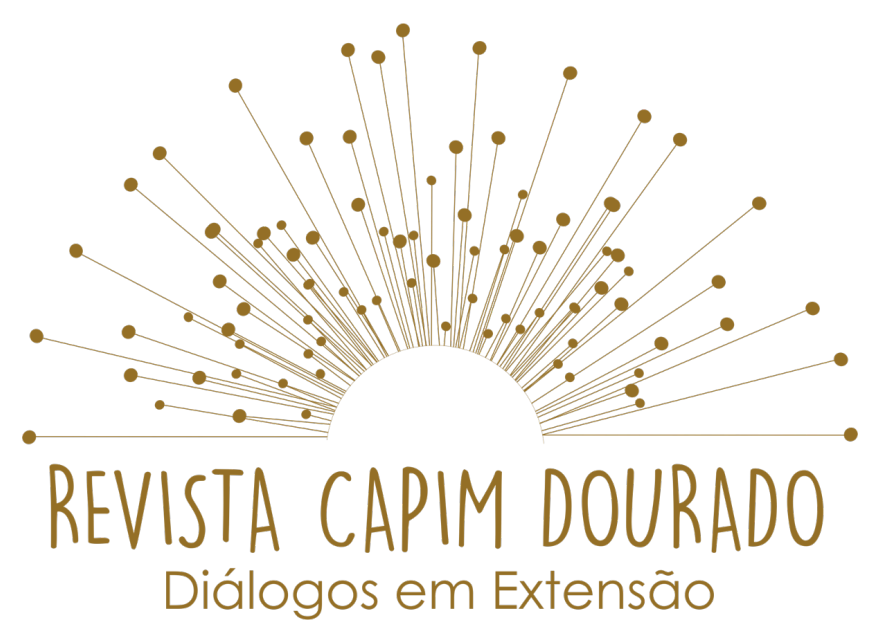

ISSN n² 2595-7341

Vol. 3, n. 1, Janeiro-Abril, 2020

DOI: http://dx.doi.org/10.20873/uft.2595-7341.2020v3n1p31

\section{INTRODUÇÃO}

O Programa de Educação Tutorial (PET) é um programa que tem como objetivo realização de atividades extracurriculares pelos acadêmicos, com ajuda de um tutor. Suas ações são ligadas a três atividades, que são ensino, pesquisa e extensão, esses pilares são de grande importância para a formação acadêmica que compõe o programa (BRASIL, 2002).

O Lar Batista F.F. Soren, que funciona desde 28/04/42, inicialmente na cidade de Itacajá/TO, abriga em casas lares crianças e adolescentes em situação de vulnerabilidade social, encaminhadas pelo Juizado da Infância e da Juventude e Conselho Tutelar. Projetos em diversas áreas temáticas são desenvolvidos com o apoio de parceiros, incluindo igrejas, universidades, empresas, instituições de saúde e profissionais voluntários (MISSÕES NACIONAIS, nd).

Assim, o objetivo deste projeto intitulado UFT além dos muros é de transferir o conhecimento e tecnologia adquiridos na universidade em forma de uma consultoria voluntária ao Lar Batista, especificamente a cozinha e dispensa do Lar que recebem diariamente doações de alimentos para o sustento de todas as crianças e adolescentes que ali residem, desta forma acreditamos que a universidade cumpre seu papel com a sociedade e comunidade local.

\section{METODOLOGIA}

Primeiramente, foram realizadas reuniões para debater a forma como seriam executadas as atividades e distribuição das tarefas. Em seguida, foi realizada uma visita técnica ao Lar Batista F.F Soren, com o intuito de fazer um 


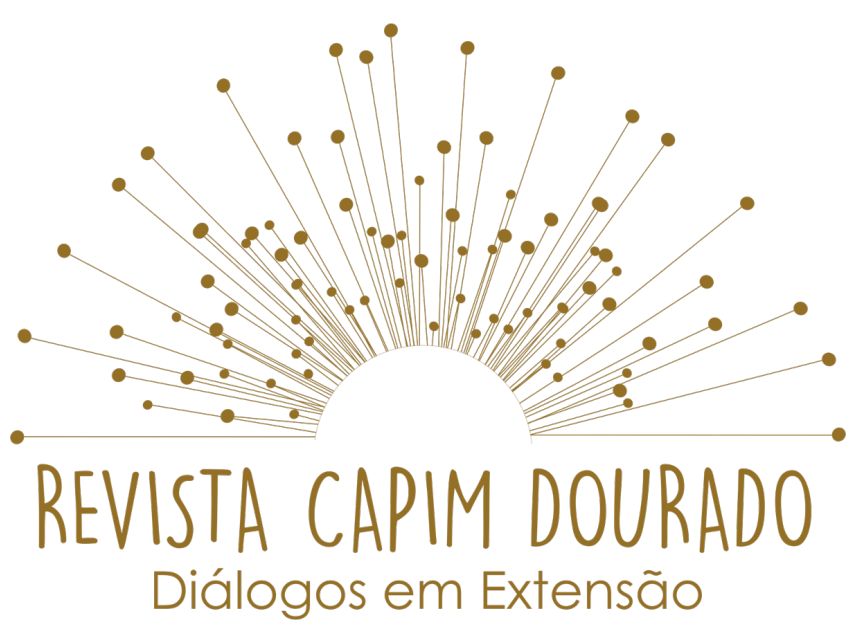

ISSN n² 2595-7341

Vol. 3, n. 1, Janeiro-Abril, 2020

DOI: http://dx.doi.org/10.20873/uft.2595-7341.2020v3n1p31

levantamento das necessidades do local avaliando as condições de armazenamentos dos alimentos nos freezers e dispensas e a forma como eram distribuídos.

Após fazer o levantamento, fez-se um planejamento das ações e tempo necessário para a execução das tarefas. Deu-se início à execução das tarefas na segunda visita ao local, onde a rotina e funcionamento da cozinha foi descrita pelos responsáveis da instituição desde a chegada e armazenamento dos alimentos até seu consumo final. A rotina descrita foi essencial para a organização dos freezers e dispensa e possível instrução aos responsáveis pelo local a forma correta na qual os alimentos deveriam ser armazenados, visando um melhor aproveitamento do espaço e dos alimentos, garantindo maior segurança aos produtos.

\section{FUNDAMENTAÇÃO TEÓRICA}

A Constituição da República Federativa do Brasil, artigo 207, consagra o princípio da indissociabilidade entre ensino, pesquisa e extensão entre as atividades universitárias (BRASIL, 1988), pilares também defendidos e eficazmente aplicados pelo PET, instituído pelo Artigo 12 da Lei no 11.180, de 23 de setembro de 2005. A indissociabilidade vem também em congruência com a Política Nacional de Extensão (PNE) que tem diretrizes baseadas em cinco eixos: impacto e transformação; interação dialogada; interdisciplinaridade; indissociabilidade entre ensino, pesquisa e extensão; impacto na formação acadêmica (FORPROEX, 1987). 


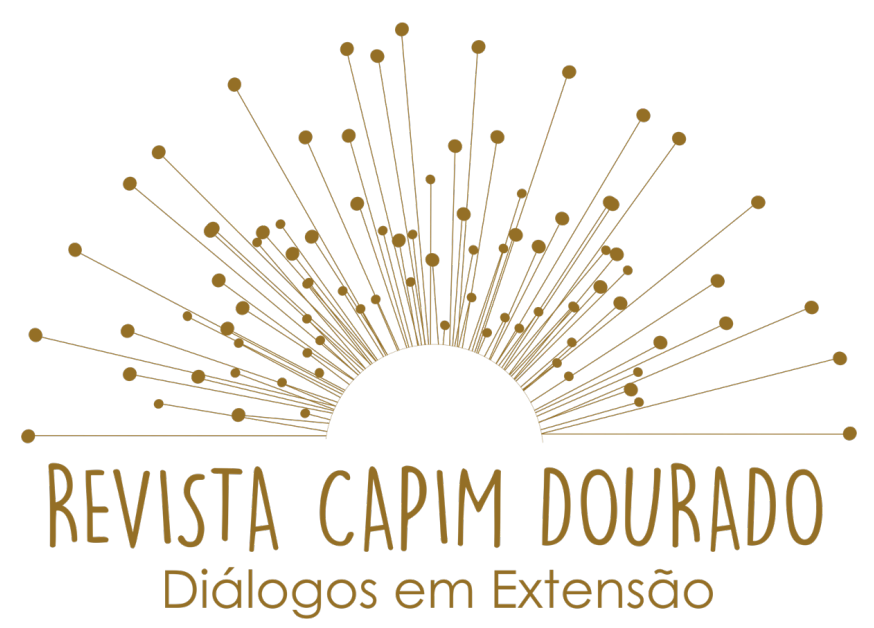

ISSN n² 2595-7341

Vol. 3, n. 1, Janeiro-Abril, 2020

DOI: http://dx.doi.org/10.20873/uft.2595-7341.2020v3n1p31

Atividades de extensão que contemplam tais diretrizes podem ter impacto transformador tanto para a sociedade quanto para a comunidade acadêmica que desenvolve e compartilha essas ações. O desenvolvimento de atividades em que a aplicação prática de saberes teóricos atende necessidades sociais impactando e transformando a realidade, além de contribuir imensamente para a formação acadêmica.

Dessa forma, assim como todas as atividades desenvolvidas e executas pelo grupo do PET- Engenharia de Alimentos, esse projeto se fundamenta nos pilares que sustentam a formação do programa.

\section{RESULTADOS FINAIS}

Uma vez tido conhecimento da existência do Lar Batista e de sua filosofia buscou-se atender a instituição de modo a aplicar os conhecimentos teóricos como forma de suprir as impertinências encontradas no local, referentes ao armazenamento dos alimentos recebidos. Sabendo-se que a instituição possui natureza filantrópica, educacional e social que acolhe crianças e adolescentes em situação de vulnerabilidade e que, por isso, recebe muitas doações de alimentos e alimenta muitas pessoas por dia, especialmente em datas comemorativas, foi notória a necessidade de um controle dos alimentos recebidos bem como seu armazenamento aplicando boas práticas de fabricação e controle de qualidade.

O PET pôde vivenciar a prática da consultoria tanto quanto interagir com a 


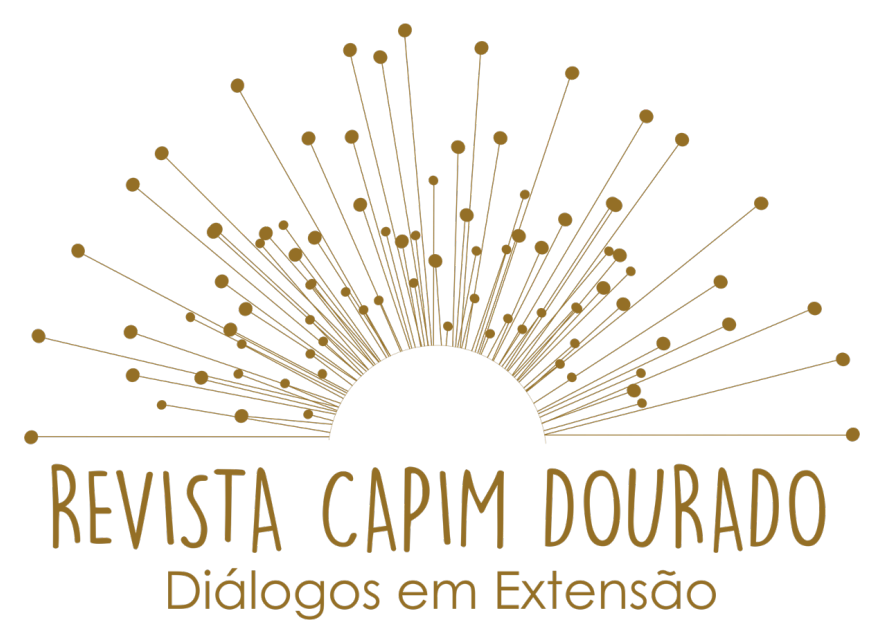

ISSN n² 2595-7341

Vol. 3, n. 1, Janeiro-Abril, 2020

DOI: http://dx.doi.org/10.20873/uft.2595-7341.2020v3n1p31

comunidade local conhecendo as propostas da instituição e as crianças e adolescentes que ali residiam.

A instituição estava aberta a sugestões e, dessa forma, inicialmente foi feito um check-list do local por intermédio de um manual de vigilância e foram apontadas duas inadequações que mereciam urgência. A primeira diz respeito a um problema com caruncho na dispensa e a segunda à organização dos freezers que, devido ao volume de doações, estava sendo feita de forma inadequada impedindo que os alimentos fossem consumidos na data de validade correta. Para o problema específico da dispensa foi sugerido, a princípio, descartar os alimentos contaminados e fazer uma faxina completa no ambiente posteriormente o caso seria mais bem estudado para que houvesse melhor solução. Quanto aos freezers, foi feita uma organização em conjunto otimizando os espaços, separando em porções menores, e etiquetando quanto ao tipo de alimento e validade priorizando para que as carnes não entrem em contato com o restante dos alimentos e alguns alimentos que não atendiam aos critérios estabelecidos foram descartados.

Toda a ação foi feita com base na Resolução-RDC n 216/2004 que rege sobre as Boas Práticas para Serviços de Alimentação fazendo uso do Art. 5 e Art. 37 para armazenamento de alimentos congelados garantindo a qualidade dos alimentos. Ao final da ação foi feito um controle, em forma de planilha do Excel, contendo nome do alimento, validade e local de armazenamento com a finalidade de facilitar a identificação da data de consumação. O PET ficou responsável por fazer visitas periódicas para que a planilha esteja sempre atualizada bem como forma de verificar se o controle está sendo feito e entrar 


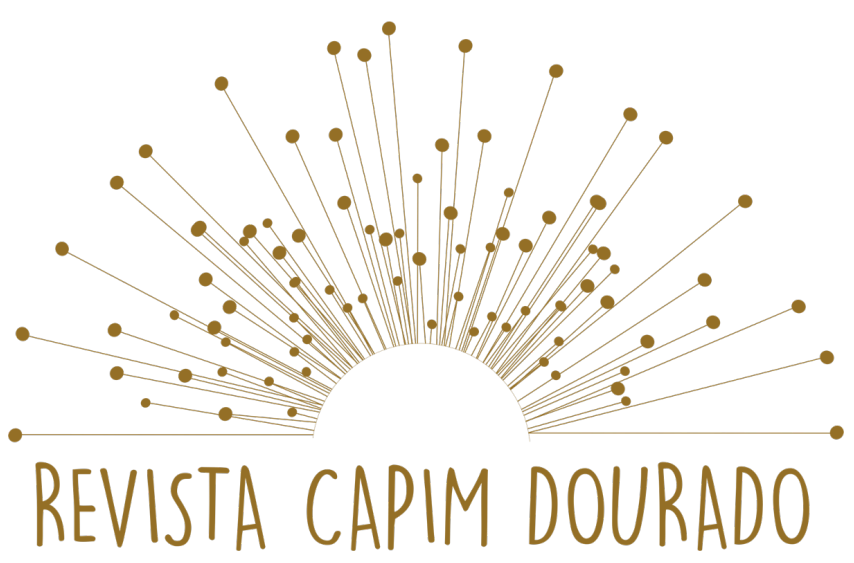

\section{Diálogos em Extensão}

ISSN n² 2595-7341

Vol. 3, n. 1, Janeiro-Abril, 2020

DOI: http://dx.doi.org/10.20873/uft.2595-7341.2020v3n1p31

em contato sempre que houver um alimento se aproximando da data de validade.

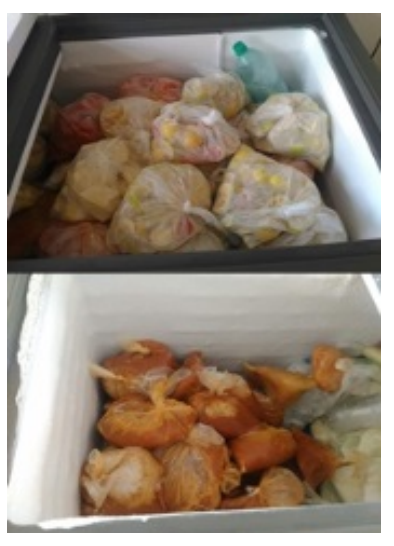

Figura 1 - Freezer antes da ação

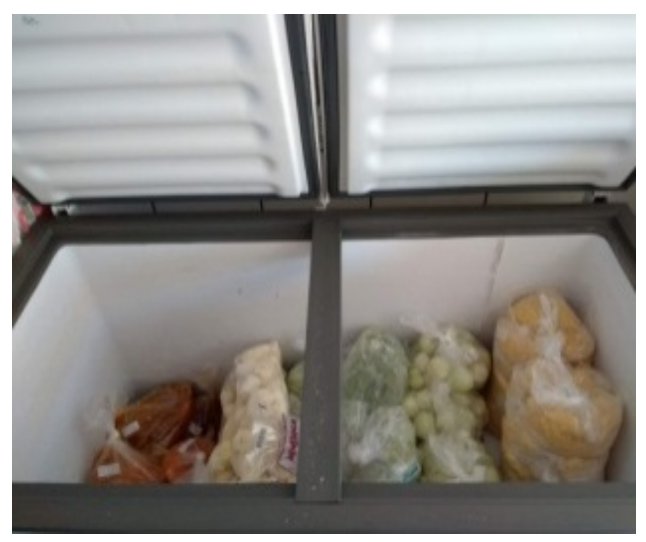

Figura 2 - Freezer após a ação

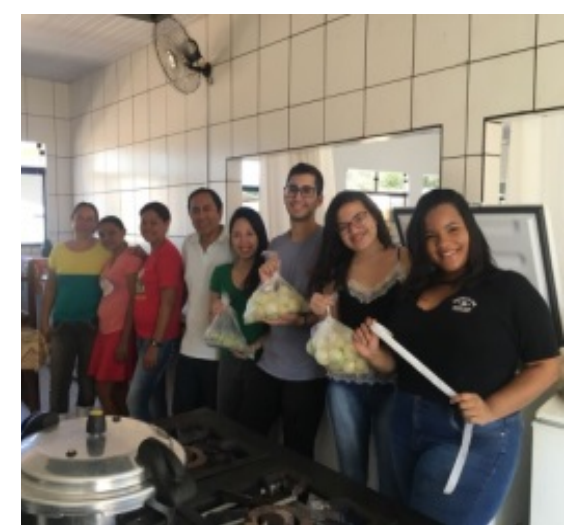

Figura 3 - Fim da ação

\section{CONSIDERAÇÕES FINAIS}

Com a análise das atividades realizadas no Lar e os resultados obtidos, pode-se afirmar que a consultoria voluntária teve êxito, permitindo a aplicação dos conhecimentos obtidos em sala de aula em áreas de interesse do curso, além de proporcionar o desenvolvimento da cidadania e consciência social aos envolvidos. A necessidade de acompanhamento supervisório mesmo após as 


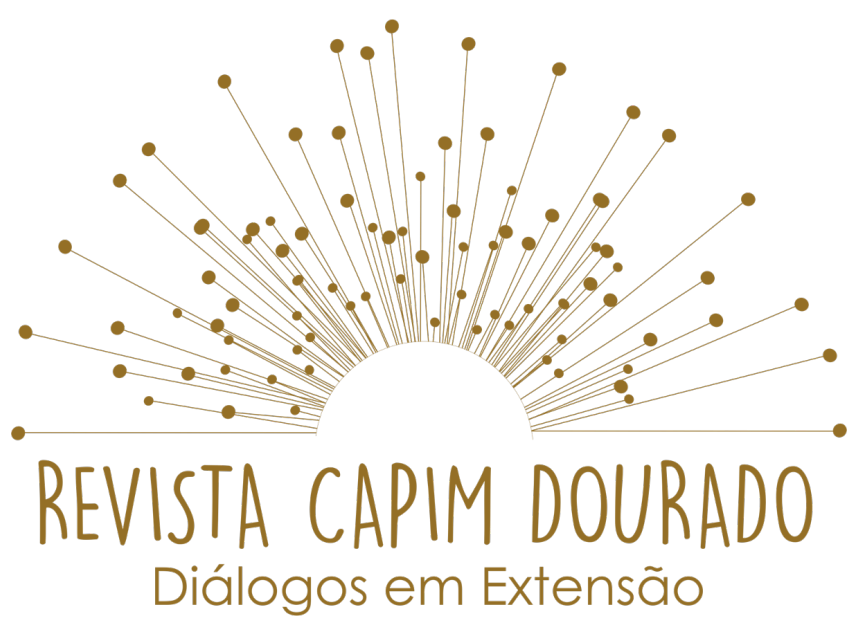

ISSN n² 2595-7341

Vol. 3, n. 1, Janeiro-Abril, 2020

DOI: http://dx.doi.org/10.20873/uft.2595-7341.2020v3n1p31

atividades propostas serem concluídas permite ainda o contínuo exercício da interdisciplinaridade não apenas dos membros desta atividade, mas de todo o grupo PET.

\section{REFERÊNCIAS}

AGENCIA NACIONAL DE VIGILÂNCIA SANITÁRIA. RDC N²16/2004: Boas Práticas para Serviços de Alimentação, 2004.

BRASIL. Constituição da República Federativa do Brasil de 1988. Brasília, 5 out 1988. Disponível em:

<http://www.planalto.gov.br/ccivil_03/constituicao/constituicao.htm>. Acesso em: 08 de agosto de 2019.

BRASIL. Manual de Orientações Básicas PET. 2002. Disponível em: $<$ http://portal.mec.gov.br/sesu/arquivos/pdf/PETmanual.pdf $>$. Acesso em: $27 \mathrm{de}$ out. 2018.

FORPROEX. Fórum De Pró-Reitores de Extensão das Universidades Públicas Brasileiras. Política Nacional de Extensão Universitária. Manaus, AM, maio 2012.

Disponivel em: <http://www.renex.org.br/documentos/2012-07-13PoliticaNacional-de-Extensao.pdf>. Acesso em: 07 de agosto de 2019.

GONÇALVES, N. G. Indissociabilidade entre Ensino, Pesquisa e Extensão: um princípio necessário. 2015. Disponível em:

https://periodicos.ufsc.br/index.php/perspectiva/article/viewFile/2175795X.2015 v33n3p1229/pdfa. Acesso em 07 de agosto de 2019. 


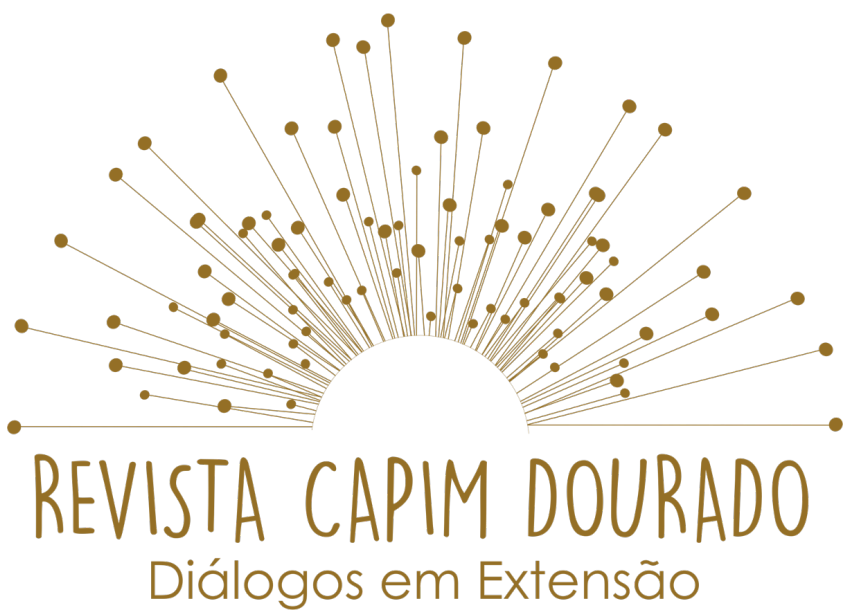

ISSN n² 2595-7341 Vol. 3, n. 1, Janeiro-Abril, 2020

DOI: http://dx.doi.org/10.20873/uft.2595-7341.2020v3n1p31

MISSÕES NACIONAIS. Lar Batista FF Soren. nd. Disponível em: <https://www.missoesnacionais.org.br/lar-batista-ff-soren>. Acesso em: 27 de outubro de 2018. 